\title{
Gerontology
}

\section{Depression and Dementia in Older People (CD-ROM)}

P.W. Overstall (ed.)

Kiss of Life Multimedia Ltd (UK)

GBP 75.00 (single user), GBP 125.00 (institutional)

This CD-ROM is a partly interactive tutorial on dementia and depression in older people. As it says on the cover, there are about five hours of tutorial study and four hours of reading material. However, these times presuppose no knowledge of the subject whatsoever. Those with some knowledge may skim a bit more and shorten the experience.

Some of the contributors are distinguished in their fields - and it shows. With the others it is easier to take issue. There are certainly some statements that are wrong. For instance, it is advised that an aggressive patient with dementia should be given medication straight away, and risperidone at that. If the aggression is due to some hitherto unrevealed physical illness, then risperidone will not do much good. Furthermore, this disk was published after the UK Committee on Safety of Medicine's advice that risperidone should not be used in patients with dementia. This is referred to elsewhere, so why recommend it (without caveat)? Another extraor- dinary statement is that the police should be informed if a psychotic patient is expressing persecutory ideas that might involve them. I should add I have never done such a thing and do not intend to start now.

The blurb states that this disk will be of 'particular interest to trainees in geriatric medicine and old age psychiatry as well as GP's and social services professionals'; so I gave it to some trainees in psychiatry and geriatric medicine to see what they thought. Opinions were generally favourable with the case scenarios and the patient interviews singled out for praise. Some of the trainees did not find the disk very user-friendly. They could not understand why mirtazepine is plugged as an antidepressant, and nor could I. As far as I can tell, Organon was not involved in the disk's production.

What concerns me most about this disk is what it represents in comparison to its competitor, namely that old-fashioned thing, the printed word. For me the disk is a dumbing-down, which fits, of course, the spirit of the age. Books and comprehensive reviews can present the subtleties, contradictions and shades of grey that are nearer to the truth, whereas such disks as this are like army field manuals - they teach the private soldier what to do without actually having to think.

Robin Jacoby, Oxford 